\title{
Dynamics of fish diversity across an environmental gradient in the Seribu Islands reefs off Jakarta
}

\author{
HAWIS H. MADDUPPA ${ }^{1, \vartheta}$, BEGINER SUBHAN ${ }^{1}$, ENY SUPARYANI ${ }^{2}$, ACHIS M. SIREGAR ${ }^{1}$, \\ DONDY ARAFAT $^{1}$, SUKMARAHARJA A. TARIGAN $^{1}$, ALIMUDDIN ${ }^{1}$, DENNY KHAIRUDI ${ }^{1}$, \\ FADHILLAH RAHMAWATI ${ }^{1}$, ADITYA BRAMANDITO ${ }^{1}$ \\ ${ }^{1}$ Laboratory of Marine Biodiversity and Biosystematics, Department of Marine Science and Technology Faculty of Fisheries and Marine Science \\ Bogor Agricultural University. Jl. Agatis No. 1, Bogor 16680, West Java, Indonesia. Tel./Fax. +62 251 8623644, ”e-mail: hawis@ipb.ac.id. \\ ${ }^{2}$ Office of Marine and Agriculture, Jakarta Province. Jl Gunung Sahari No XI, Jakarta Pusat 10720, Jakarta, Indonesia
}

Manuscript received: 19 March 2013. Revision accepted: 17 April 2013.

\begin{abstract}
Madduppa HH, Subhan B, Suparyani E, Siregar AM, Arafat D, Tarigan SA, Alimuddin, Khairudi D, Rahmawati F, Bramandito A. 2013. Dynamics of fish diversity across an environmental gradient in the Seribu Islands reefs off Jakarta. Biodiversitas 14: 17-24. The reefs of Seribu Islands have been affected by multitude of anthropogenic pressures. However, the biodiversity of reef fishes across the archipelago linked to environmental condition is poorly known. This study aimed to investigate the biodiversity and the trophic level of fish communities across the archipelago. The study on reef fish communities was conducted on 33 reef sites associated with islands or shoal randomly chosen from each zone along environmental gradients from the inshore water nearest of Jakarta Bay to the offshore water of the outer islands. The study sites represented each sub-districts within the archipelago, namely Pari, Tidung, Panggang, Kelapa, and Harapan. A total of 46,263 individual fishes were counted, belonging to 216 species and 29 families. The multivariate analysis of fish abundance using the Bray Curtis similarity index and non-metric multidimensional scaling (MDS) clearly showed the clustering of sub-districts, near and far from Jakarta Bay. The results showed that the sub-districts can be clustered into three groups. Group one consists of one sub-district (Pari) located in the southern part of the Seribu Islands near Jakarta Bay. Group two consists of three subdistricts (Tidung, Panggang, Kelapa) located in mid of the archipelago. The third group consists of one sub-district (Harapan) located in the northern part of the Seribu Islands. Based on species richness and fish diversity indices, the sub-districts can be clustered into two groups $(1$ = Pari and Tidung, 2 = Panggang Kelapa, Harapan). However, levels of similarities among sub-districts varied. The fish community in sub-district of Pari was dominated by carnivorous, omnivorous and herbivorous fishes, while those in the rest of subdistricts were dominated by omnivorous and carnivorous fishes. The present study results showed that the biodiversity of reef fishes across the Seribu Islands seemed to be linked to the environmental conditions.
\end{abstract}

Key words: Fish-habitat association, species diversity, anthropogenic stress, multivariate analysis

\section{INTRODUCTION}

Coral reefs are heavily influenced by the human activities through pollution and habitat loss throughout the world (Burke et al. 2011), and sea level rise or the increase of ocean temperature due to the global change (Hughes et al. 2003). In Indonesia, marine communities have been impacted by an increase in eutrophication and sedimentation levels as shown in the waters of Jakarta Bay (Verstappen 1988; Marques et al. 1997; Renema 2008). As a result of increased sedimentation, nutrient loading, and chemical contamination, coral reefs became degraded (Rees et al. 1999; Williams et al. 2000). Furthermore, reef degradation could affect the coral reef fish communities due to their strong relationship.

The Seribu Islands (or Thousand Islands, Kepulauan Seribu), which consists of 110 islands spread from the Jakarta Bay to as far as $80 \mathrm{~km}$ to the north of the Java Sea, have been threatened by different kinds of anthropogenic pressures including coral mining, fishing, anchor damage, oil spills, resort construction and the discharge of industrial and domestic effluents (Rees et al. 1999; Rachello-Dolmen and Cleary 2007; Willoughby 1986; Uneputty and Evans 1997). In the 1980s, the archipelago reefs also experienced bleaching phenomenon due to ENSO (El Niño Southern Oscillation) resulting in the death of mainly branching species of the genera of Acropora and Pocillopora (Brown and Suharsono 1990).

Regions of the Seribu Islands are divided into three zones according to environmental gradient from the inshore water of Jakarta Bay to the offshore water of the outer islands (Hutomo and Adrim 1985). Since reef studies in 1920 s, the reefs surrounding Onrust Island, located in Jakarta Bay, have been excluded from reef studies due to measurable anthropogenic influences (Zaneveld and Verstappen 1952). The environmental pressures on Jakarta Bay have increased until today and have been noted in several studies (e.g. Tomascik et al. 1997). A number of studies also show that reef coverage in Jakarta Bay is very low and shifts toward the Seribu Islands, as a result of diminishing human activities and pollution (Verstappen 1988; Cleary et al. 2006). 
The gradient of environmental quality has changed the marine biodiversity across Seribu Islands, such as sponges (de Voogd and Cleary 2008), mollusk (van der Meij et al. 2009) and corals (Cleary et al. 2006). Complexity of coral reefs and spatial variability affect the trophic structure of the fish community. For instance, the decrease of live corals has increased the coverage of algae which in turn gives benefit to herbivorous fishes (Madduppa et al. 2012). Therefore, the current study aimed to investigate the biodiversity dynamics and the trophic levels of fish communities across the archipelago.

\section{MATERIALS AND METHODS}

\section{Study sites}

The Seribu Islands Marine National Park has been declared as a National Reserve in 1982 (Uneputty and Evans 1997). Since 2006, the Seribu Islands is administratively divided into two districts (Estradivari et al. 2007). First, The District of North Seribu Islands which covers 79 islands within three sub-districts i.e. Kelapa (36 islands), Harapan (30), and Panggang (13). Second, The District of South Seribu Islands which is divided into three sub-



Figure 1. Location of the Seribu Islands, north of Jakarta, Java Island, Indonesia. The map at the upper right shows the position of Seribu Islands relative to Indonesia. The sampling sites indicated by flag. 
districts covering 31 islands. The Seribu Islands Marine National Park which covers an area of 107.489 ha or approximately $20 \%$ of the total region. Two different seasons are affecting the Seribu Islands, namely 'wet' season (November-March) during the northwest monsoon, and 'dry' season (May-September) during the southeast monsoon (Rees et al. 1999). Figure 1 shows the study sites at Seribu Islands.

The study on reef fish communities was conducted at 33 reef sites associated with islands or shoal which were randomly chosen from each zone along environmental gradients from the inshore water nearest of Jakarta Bay to the offshore water of the outer islands and represented each sub-districts within the archipelago, namely Pari (Lancang Is., Bokor Is.), Tidung (Tidung Besar Is., Payung Besar Is., Karang Beras), Panggang (Karang Bongkok, Gosong Air, Kotok Kecil Is., Karang Congkak, Sekati Is., Semak Daun Is., Air Barat Is., Kotok Besar Is.), Kelapa (Genteng Is., Jukung Is., Kaliage Besar Is., Kaliage Kecil Is., Kayu Angin Semut Is., Kelapa Is., Lipan Is., Malinjo Is., Matahari Is., Melintang Is., Satu Is., Semut Besar Is., Semut Timur Is.), and Harapan (Opak Besar Is., Opak Kecil Is., Sepa Besar Is., Sepa Kecil Is.). Two different depths $(3$ and $10 \mathrm{~m}$ ) for each sampling site at reef slope were selected (English et al. 1997).

\section{Data collection}

Sampling was carried out at each site between 09.00 and 16.30, November 10-20, 2011 during a coral reef expedition by Marine Biology Laboratory, Bogor Agricultural University. Reef fish communities were assessed by underwater visual census (UVC) on a transect line of 50 meters at each depth (English et al. 1997). In an attempt to reduce daily variability of fish density data (caused by differences in nocturnal and diurnal behavior), sampling excluded the high activity periods of early morning and late afternoon (Colton and Alevizon 1981; English et al. 1997). During each census, the observer waited for 5 to 10 minutes before beginning the data recording along transect in order to allow the fishes to resume their normal behaviours (Brock 1982; Halford and Thompson 1994). Only individuals within $2.5 \mathrm{~m}$ on either side and $5 \mathrm{~m}$ above along the transect, were counted. Each individual (cryptic and large pelagic species were excluded) was counted and identified to species level. In order to avoid the influence of temporal recruitment events, fish recruits up to a size of $\sim 3-5 \mathrm{~cm}$ were excluded from the count. After data collection, reef fish identification was confirmed by using standard fish identification books (i.e. Allen 2000; Kuiter 1992). The trophic level for each species was confirmed with the Fishbase (Froese and Pauly 2010).

\section{Data analysis}

The community Shannon-Wiener diversity index $\mathrm{H}^{\prime}$ was calculated on a natural logarithm (ln) basis (Magurran 1988; Shannon and Weaver 1949). Poisson regression analysis was used to test the statistical significance of differences in fish abundance among sites (sub districts), as

well diversity and species richness, using statistical package STATISTICA 7.0.

Multivariate analysis of the fish community data were conducted using the program PRIMER 5.2.9 (Clarke and Gorley 2001; Kruskal 1964). Fish abundance, species richness, and species diversity data were fourth-root transformed prior to analysis to reduce the influence of some overlay abundant species and give more weight to rare species while retaining the information value of relative abundances, an approach frequently used in the multivariate analysis of community data (Clarke and Green 1988; Field et al. 1982).

Bray-Curtis similarity and Non-metric Multidimensional Scaling (MDS) were performed to visualize differences in fish communities from the different sites (Kruskal 1964; Shepard 1962). MDS was based on Bray-Curtis similarities, and 100 restarts were used for the calculations.

\section{RESULTS AND DISCUSSION}

A total of 46,263 individual fishes were counted, belonging to 216 species and 29 families (Table 1). A total of 49 and 78 fish species were recorded from sub-districts of Pari and Tidung, 109 and 148 from sub-districts of Panggang and Kelapa, and 106 from sub-district of Harapan. The values of species richness in this study were almost similar to that observed by Estradivari et al. (2007) in 2004-2005 in the Seribu Islands (211 species). In addition, the species richness of the Seribu Islands were also similar in range to those observed at other Indonesian coral reefs, such as Togean Islands and Weh Island (Allen and Werner 2002). The low species richness in the subdistricts of Seribu Islands near from Jakarta Bay (e.g. Pari and Tidung) might be related to the pressures on the environment such as bleaching resulting from the 1982/83 ENSO event (Brown and Suharsono 1990; Hoeksema 1991), and anthropogenic factors such as land-based contaminants, man-made objects, oil pollution, domestic and industrial refuse (Willoughby 1986; Uneputty and Evans 1997; Rees et al. 1999).

Table 1. Total number and trophic level of fish species at each subdistrict in the Seribu Islands

Family

Species

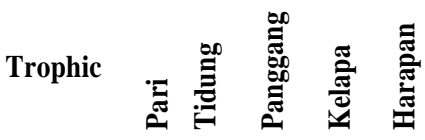

Acanthuridae

Acanthurus sp.

Ctenochaetus striatus

Apogonidae

Apogon angustatus

Apogon apogonides

Apogon aureus

Apogon cavitienis

Apogon chrysopomus

Apogon compressus

Apogon novemfasciatus

Apogon sealei

Apogon semiornatus

\begin{tabular}{llllll} 
Herbivore & 0 & 1 & 1 & 0 & 0 \\
Omnivore & 0 & 1 & 0 & 4 & 0 \\
& & & & & \\
Carnivore & 0 & 0 & 0 & 7 & 0 \\
Carnivore & 0 & 0 & 80 & 23 & 0 \\
Carnivore & 0 & 0 & 11 & 37 & 89 \\
Carnivore & 0 & 0 & 20 & 0 & 0 \\
Carnivore & 0 & 13 & 0 & 0 & 0 \\
Carnivore & 2 & 20 & 331 & 293 & 192 \\
Carnivore & 0 & 0 & 24 & 0 & 43 \\
Carnivore & 20 & 0 & 0 & 0 & 0 \\
Carnivore & 0 & 0 & 17 & 0 & 2 \\
\hline
\end{tabular}




\begin{tabular}{|c|c|c|c|c|c|c|c|c|c|c|c|c|c|}
\hline Archamea fucata & Carnivore & 0 & 0 & 3 & 0 & 0 & Halichoeres leucurus & Omnivore & 4 & 13 & 45 & 58 & 39 \\
\hline Cheilodipterus artus & Carnivore & 0 & 0 & 52 & 33 & 88 & Halichoeres marginatus & Omnivore & 0 & 4 & 15 & 41 & 22 \\
\hline Cheilodipterus isostigmus & Carnivore & 2 & 0 & 0 & 0 & 0 & Halichoeres melanochir & Omnivore & 0 & 0 & 28 & 48 & 5 \\
\hline Cheilodipterus sp. & Carnivore & 0 & 0 & 0 & 24 & 0 & Halichoeres melanurus & Omnivore & 3 & 11 & 67 & 47 & 29 \\
\hline Sphaeramia nematoptera & Carnivore & 0 & 0 & 0 & 22 & 0 & Halichoeres nigrescens & Carnivore & 14 & 2 & 2 & 1 & 0 \\
\hline Aulostomidae & & & & & & & Halichoeres ornatissimus & Carnivore & 0 & 0 & 12 & 30 & 0 \\
\hline Aulostomus chinensis & Carnivore & 0 & 0 & 0 & 3 & 0 & Halichoeres richmondi & Carnivore & 0 & 9 & 28 & 22 & 11 \\
\hline Balistidae & & & & & & & Halichoeres scapularis & Carnivore & 1 & 0 & 0 & 0 & 0 \\
\hline Balistoides sp. & Carnivore & 0 & 1 & 0 & 0 & 0 & Halichoeres sp. & Omnivore & 6 & 8 & 74 & 8 & 1 \\
\hline Melichthys indicus & Omnivore & 0 & 0 & 7 & 0 & 2 & Halichoeres vrolikii & Omnivore & 5 & 0 & 0 & 1 & 0 \\
\hline Caesionidae & & & & & & & Hemigymnus melapterus & Carnivore & 3 & 4 & 10 & 26 & 4 \\
\hline Caesio cuning & Planktivore & & 0 & 429 & 209 & 116 & Labroides chrysotaenia & Omnivore & 0 & 0 & 0 & 0 & 2 \\
\hline Caesio teres & Planktivore & & 260 & 372 & 906 & 475 & Labroides dimidiatus & Carnivore & 6 & 16 & 44 & 61 & 27 \\
\hline Pterocaesio digramma & Carnivore & 0 & 0 & 0 & 0 & 50 & Macropharyngodon negrosensis & Omnivore & 0 & 0 & 2 & 7 & 8 \\
\hline Pterocaesio tile & Planktivore & 0 & 0 & 38 & 80 & 0 & Neoglyphidodon melas & Omnivore & 0 & 0 & 0 & 0 & 0 \\
\hline Centriscidae & & & & & & & Pseudocheilinus hexataenia & Omnivore & 0 & 0 & 5 & 7 & 11 \\
\hline Aeoliscus strigatus & Carnivore & 4 & 2 & 0 & 24 & 16 & Pseudojuloides cerasinus & Omnivore & 0 & 0 & 18 & 0 & 18 \\
\hline Chaetodontidae & & & & & & & Pteragogus amboinensis & Omnivore & 0 & 0 & 0 & 0 & 22 \\
\hline Chaetodon collare & Herbivore & 1 & 0 & 0 & 0 & 0 & Stethojulis trilineata & Omnivore & 0 & 11 & 0 & 0 & 3 \\
\hline Chaetodon meyeri & Herbiivore & 0 & 0 & 5 & 5 & 0 & Thalassoma lunare & Omnivore & 24 & 78 & 132 & 195 & 45 \\
\hline Chaetodon octofasciatus & Coralivore & 0 & 57 & 131 & 196 & 66 & Thalassoma lutescens & Omnivore & 0 & 2 & 0 & 0 & 5 \\
\hline Chelmon rostratus & Coralivore & 0 & 0 & 3 & 9 & 2 & Thalassoma purpureum & Carnivore & 0 & 0 & 11 & 7 & 22 \\
\hline Chaetodon melanopus & Coralivore & 0 & 0 & 0 & 0 & 2 & Thalassoma quinquevittatum & Carnivore & 0 & 0 & 16 & 0 & 19 \\
\hline Coradion trifasciatus & Coralivore & 0 & 0 & 3 & 0 & 6 & Lethrinidae & & & & & & \\
\hline Heniochus chrysostomus & Coralivore & 0 & 1 & 0 & 0 & 0 & Lethrinus erythropterus & Carnivore & 0 & 0 & 0 & 12 & 0 \\
\hline Heniochus pleurotaenia & Coralivore & 0 & 3 & 2 & 0 & 4 & Lutjanidae & & & & & & \\
\hline Heniochus varius & Coralivore & 0 & 0 & 11 & 7 & 1 & Lutjanus biguttatus & Carnivore & 0 & 2 & 4 & 26 & 0 \\
\hline Cirrhitidae & & & & & & & Lutjanus decussatus & Carnivore & 0 & 6 & 25 & 25 & 3 \\
\hline Paracirrhites sp. & Carnivore & 1 & 0 & 0 & 0 & 0 & Lutjanus kasmira & Omnivore & 0 & 0 & 0 & 6 & 0 \\
\hline Dasyatidae & & & & & & & Lutjanus russellii & Carnivore & 0 & 0 & 0 & 4 & 0 \\
\hline Taeniura lymma & Carnivore & 0 & 0 & 1 & 1 & 0 & Mullidae & & & & & & \\
\hline Echeneidae & & & & & & & Parupeneus barberinus & Carnivore & 0 & 0 & 0 & 5 & 0 \\
\hline Remora sp. & Omnivore & 2 & 0 & 0 & 0 & 0 & Muraenidae & & & & & & \\
\hline Ephippidae & & & & & & & Gymnothorax javanicus & Carnivore & 0 & 0 & 0 & 1 & 0 \\
\hline Platax pinnatus & Omnivore & 0 & 0 & 2 & 0 & 0 & Nemipteridae & & & & & & \\
\hline Platax teira & Omnivore & 1 & 2 & 2 & 3 & 0 & Pentapodus caninus & Carnivore & 0 & 0 & 46 & 28 & 28 \\
\hline Gobiidae & & & & & & & Pentapodus sp. & Carnivore & 0 & 0 & 0 & 1 & 0 \\
\hline Exyrias belissimus & Omnivore & 0 & 0 & 0 & 1 & 0 & Pentapodus vitta & Carnivore & 0 & 0 & 0 & 18 & 0 \\
\hline Istigobius decorates & Omnivore & 0 & 0 & 7 & 8 & 1 & Scolopsis bilineatus & Carnivore & 3 & 22 & 29 & 50 & 17 \\
\hline Haemulidae & & & & & & & Scolopsis ciliatus & Carnivore & 0 & 3 & 0 & 9 & 0 \\
\hline Plectorhinchus chaetodontoides & Carnivore & 0 & 0 & 0 & 2 & 0 & Scolopsis lineatus & Carnivore & 0 & 0 & 17 & 14 & 17 \\
\hline Plectorhinchus chrysotaenia & Carnivore & 0 & 0 & 8 & 0 & 0 & Scolopsis margaritifer & Carnivore & 0 & 6 & 0 & 41 & 0 \\
\hline Plectorhinchus vittatus & Carnivore & 0 & 0 & 0 & 2 & 0 & Scolopsis sp. & Carnivore & 0 & 0 & 0 & 4 & 0 \\
\hline Hemirhamphidae & & & & & & & Scolopsis temporalis & Carnivore & 1 & 0 & 0 & 0 & 0 \\
\hline $\begin{array}{l}\text { Hemirhamphus far } \\
\text { Holocentridae }\end{array}$ & Omnivore & 0 & 0 & 1 & 0 & 0 & $\begin{array}{l}\text { Scolopsis trilineatus } \\
\text { Pempheridae }\end{array}$ & Carnivore & 1 & 0 & 0 & 26 & 12 \\
\hline Myripristis berndti & Carnivore & 0 & 1 & 0 & 0 & 0 & Pempheris oualensis & Carnivore & 0 & 0 & 0 & 15 & 36 \\
\hline Myripristis sp. & Carnivore & 0 & 0 & 0 & 5 & 0 & Pempheris sp. & Carnivore & 50 & 0 & 0 & 0 & 0 \\
\hline Sargocentron diadema & Carnivore & 0 & 0 & 20 & 0 & 0 & Pomacanthidae & & & & & & \\
\hline Sargocentron rubrum & Carnivore & 0 & 2 & 0 & 0 & 0 & Centropyge vrolikii & Herbivore & 0 & 1 & 0 & 0 & 0 \\
\hline Sargocentron sp. & Carnivore & 0 & 0 & 0 & 19 & 0 & Chaetodontoplus mesoleucus & Herbivore & 0 & 19 & 57 & 199 & 69 \\
\hline $\begin{array}{l}\text { Sargocentron tiereoides } \\
\text { Labridae }\end{array}$ & Carnivore & 0 & 0 & 11 & 0 & 0 & $\begin{array}{l}\text { Pomacanthus sextriatus } \\
\text { Pomacentridae }\end{array}$ & Herbivore & 0 & 0 & 2 & 0 & 0 \\
\hline Anampses sp. & Carnivore & 0 & 0 & 0 & 1 & 0 & Abudefduf bengalensis & ore & 0 & 0 & 0 & 91 & 25 \\
\hline Bodianus mesothorax & Carnivore & 1 & 6 & 19 & 27 & 24 & Abudefduf curacao & Omnivore & 0 & 0 & 0 & 18 & 0 \\
\hline Cheilinus chlorourus & Carnivore & 0 & 0 & 0 & 7 & 0 & Abudefduf septemfasciatus & Omnivore & 3 & 0 & 19 & 0 & 0 \\
\hline Cheilinus diagramma & Carnivore & 0 & 0 & 0 & 15 & 0 & Abudefduf sexfasciatus & Omnivore & 0 & 111 & 107 & 204 & 121 \\
\hline Cheilinus fasciatus & Carnivore & 0 & 29 & 67 & 96 & 19 & Abudefduf sordidus & Omnivore & 0 & 0 & 49 & 0 & 0 \\
\hline Cheilinus hortulanus & Carnivore & 0 & 0 & 15 & 8 & 0 & Abudefduf vaigiensis & Omnivore & 0 & 94 & 119 & 139 & 61 \\
\hline Cheilinus oxyrhynchus & Carnivore & 0 & 0 & 0 & 6 & 0 & Acanthochromis polyancanthus & Omnivore & 0 & 0 & 0 & 11 & 13 \\
\hline Cheilinus trilobatus & Carnivore & 0 & 0 & 0 & 3 & 0 & Amblyglyphidodon aureus & Omnivore & 0 & 2 & 0 & 25 & 2 \\
\hline Cheilinus unifasciatus & Carnivore & 0 & 0 & 0 & 6 & 0 & Amblyglyphidodon batunai & Omnivore & 0 & 0 & 49 & 79 & 87 \\
\hline Choerodon anchorago & Carnivore & 1 & 10 & 2 & 32 & 0 & Amblyglyphidodon curacao & Omnivore & 20 & 114 & 519 & 560 & 451 \\
\hline Choerodon fasciatus & Carnivore & 0 & 0 & 4 & 25 & 23 & Amblyglyphidodon leucogaster & Omnivore & 0 & 16 & 226 & 342 & 163 \\
\hline Cirrhilabrus cyanopleura & Planktivore & 40 & 1402 & 1930 & 3782 & 1770 & Amblyglyphidodon nigroris & Omnivore & 0 & 39 & 0 & 0 & 0 \\
\hline Ctenochaetus striatus & Omnivore & 0 & 0 & 7 & 0 & 0 & Amphiprion akallopisos & Omnivore & 2 & 0 & 2 & 12 & 0 \\
\hline Diproctacanthus xanthurus & Corallivore & 1 & 16 & 2 & 27 & 52 & Amphiprion akindinos & Omnivore & 0 & 0 & 0 & 0 & 5 \\
\hline Epibulus insidiator & Carnivore & 0 & 1 & 0 & 6 & 0 & Amphiprion clarkii & Omnivore & 0 & 0 & 0 & 3 & 3 \\
\hline Gomphosus varius & Carnivore & 0 & 0 & 0 & 9 & 0 & Amphiprion ocellaris & Omnivore & 0 & 0 & 0 & 0 & 6 \\
\hline Halichoeres binotopsis & Carnivore & 0 & 5 & 0 & 2 & 1 & Amphiprion perideraion & Omnivore & 0 & 0 & 0 & 0 & 2 \\
\hline Halichoeres biocellatus & Carnivore & 0 & 0 & 18 & 39 & 19 & Amphiprion sandaricinos & Omnivore & 0 & 0 & 0 & 0 & 4 \\
\hline Halichoeres chloropterus & Carnivore & 0 & 0 & 45 & 30 & 0 & Cheiloprion labiatus & Omnivore & 0 & 0 & 0 & 3 & 0 \\
\hline Halichoeres chrysotaenia & Carnivore & 0 & 5 & 7 & 21 & 0 & Chlororus sordidus & Omnivore & 0 & 0 & 0 & 2 & 0 \\
\hline Halichoeres dussumieri & Omnivore & 0 & 0 & 21 & 0 & 53 & Chromis amboinensis & Omnivore & 0 & 90 & 50 & 93 & 94 \\
\hline Halichoeres hortulanus & Carnivore & 3 & 17 & 29 & 80 & 20 & Chromis atripectoralis & Omnivore & 0 & 39 & 708 & 500 & 878 \\
\hline
\end{tabular}




\begin{tabular}{|c|c|c|c|c|c|}
\hline Chromis fumea & Planktivore 80 & 0 & 0 & 50 & 303 \\
\hline Chromis nitida & Omnivore 0 & 0 & 0 & 5 & 0 \\
\hline Chromis scotochilopterus & Planktivore 0 & 0 & 24 & 0 & 34 \\
\hline Chromis smithi & Omnivore 0 & 0 & 0 & 17 & 0 \\
\hline Chromis ternatensis & Planktivore 14 & 52 & 2 & 926 & 712 \\
\hline Chromis viridis & Omnivore 0 & 142 & 159 & 372 & 177 \\
\hline Chromis xanthura & Planktivore 0 & 0 & 69 & 78 & 70 \\
\hline Chrysiptera cyanea & Omnivore 0 & 0 & 10 & 0 & 0 \\
\hline Chrysiptera hemicyanea & Omnivore 0 & 0 & 21 & 21 & 9 \\
\hline Chrysiptera parasema & Planktivore 0 & 0 & 30 & 22 & 32 \\
\hline Chrysiptera sp. & Omnivore 6 & 0 & 0 & 0 & 0 \\
\hline Dascyllus melanurus & Omnivore 0 & 0 & 0 & 1 & 0 \\
\hline Dascyllus reticulatus & Omnivore 0 & 0 & 0 & 1 & 0 \\
\hline Dascyllus trimaculatus & Omnivore 0 & 1 & 0 & 80 & 10 \\
\hline Diproctacanthus xanthurus & Corallivore 0 & 1 & 0 & 2 & 0 \\
\hline Dischistodus melanotus & Herbivore 0 & 0 & 47 & 48 & 35 \\
\hline Dischistodus perspicillatus & Herbivore 0 & 0 & 0 & 10 & 0 \\
\hline Dischistodus prosopotaenia & Herbivore 0 & 24 & 64 & 101 & 32 \\
\hline Hemiglyphidodon plagiometopon & Herbivore 0 & 0 & 3 & 24 & 5 \\
\hline Neoglyphidodon bonang & Omnivore 0 & 0 & 2 & 0 & 0 \\
\hline Neoglyphidodon crossi & Omnivore 0 & 25 & 167 & 58 & 94 \\
\hline Neoglyphidodon leucogaster & Carnivore 0 & 25 & 0 & 0 & 0 \\
\hline Neoglyphidodon melas & Omnivore 2 & 19 & 27 & 111 & 117 \\
\hline Neoglyphidodon nigroris & Omnivore 3 & 18 & 37 & 129 & 17 \\
\hline Neoglyphidodon thoracotaeniatus & Omnivore 0 & 0 & 0 & 88 & 0 \\
\hline Neopomacentrus anabatoides & Planktivore 0 & 0 & 0 & 38 & 0 \\
\hline Neopomacentrus bankieri & Carnivore 0 & 0 & 0 & 0 & 20 \\
\hline Neopomacentrus cyanomos & Carnivore 0 & 0 & 0 & 156 & 0 \\
\hline Neopomacentrus filamentosus & Planktivore 0 & 0 & 120 & 178 & 71 \\
\hline Pomacentrus alexanderae & Omnivore 1 & 1338 & 2320 & 4058 & 1335 \\
\hline Pomacentrus amboinensis & Omnivore 0 & 0 & 0 & 57 & 0 \\
\hline Pomacentrus brachialis & Omnivore 6 & 0 & 0 & 0 & 0 \\
\hline Pomacentrus burroughi & Herbivore 1 & 22 & 23 & 77 & 5 \\
\hline Pomacentrus coelestis & Omnivore 0 & 0 & 56 & 0 & 12 \\
\hline Pomacentrus cuneatus & Omnivore 7 & 0 & 0 & 0 & 0 \\
\hline Pomacentrus javanicus & Omnivore 0 & 14 & 0 & 0 & 0 \\
\hline Pomacentrus lepidogenys & Planktivore 13 & 33 & 33 & 514 & 98 \\
\hline Pomacentrus littoralis & Omnivore 0 & 18 & 0 & 0 & 0 \\
\hline Pomacentrus milleri & Omnivore 5 & 14 & 0 & 56 & 11 \\
\hline Pomacentrus moluccensis & Omnivore 5 & 306 & 378 & 297 & 103 \\
\hline Pomacentrus simsiang & Omnivore 0 & 0 & 0 & 16 & 0 \\
\hline Pomacentrus smithi & Omnivore 50 & 657 & 775 & 2760 & 239 \\
\hline Pomacentrus sp. & Omnivore 5 & 1 & 0 & 9 & 2 \\
\hline Pomacentrus xanthosternus & Omnivore 0 & 12 & 0 & 0 & 0 \\
\hline Premnas biaculatus & Omnivore 1 & 0 & 0 & 2 & 0 \\
\hline Pristotis obtusirostris & Omnivore 0 & 0 & 18 & 0 & 23 \\
\hline \multicolumn{6}{|l|}{ Scaridae } \\
\hline Chlorurus bleekeri & Herbivore 0 & 0 & 1 & 18 & 0 \\
\hline Chlorurus microrhinos & Herbivore 0 & 0 & 8 & 29 & 0 \\
\hline Chlorurus sordidus & Omnivore 0 & 9 & 33 & 107 & 39 \\
\hline Scarus chameleon & Herbivore 0 & 0 & 0 & 0 & 4 \\
\hline Scarus dimidiatus & Herbivore 0 & 0 & 6 & 0 & 21 \\
\hline Scarus flavipectoralis & Herbivore 0 & 0 & 6 & 0 & 18 \\
\hline Scarus frenatus & Herbivore 0 & 0 & 5 & 0 & 10 \\
\hline Scarus ghobban & Herbivore 0 & 0 & 2 & 17 & 1 \\
\hline Scarus globiceps & Herbivore 0 & 2 & 5 & 7 & 18 \\
\hline Scarus niger & Herbivore 2 & 7 & 27 & 37 & 5 \\
\hline Scarus quoyi & Herbivore 0 & 8 & 8 & 8 & 0 \\
\hline Scarus rivulatus & Herbivore 0 & 42 & 17 & 35 & 13 \\
\hline Scarus sordidus & Herbivore 0 & 0 & 0 & 5 & 0 \\
\hline Scarus sp. & Herbivore 2 & 26 & 0 & 11 & 1 \\
\hline Scarus xanthopleura & Herbivore 0 & 0 & 0 & 5 & 0 \\
\hline \multicolumn{6}{|l|}{ Scorpaenidae } \\
\hline Pterois volitans & Carnivore 0 & 0 & 0 & 1 & 0 \\
\hline \multicolumn{6}{|l|}{ Serranidae } \\
\hline Cephalopholis argus & Carnivore 0 & 0 & 0 & 26 & 0 \\
\hline Cephalopholis boenak & Carnivore 4 & 1 & 0 & 13 & 0 \\
\hline Cephalopholis microprion & Carnivore 1 & 4 & 4 & 16 & 2 \\
\hline Cephalopholis sp. & Carnivore 1 & 0 & 0 & 1 & 0 \\
\hline Diploprion bifasciatum & Carnivore 0 & 1 & 0 & 0 & 0 \\
\hline Epinephelus fasciatus & Carnivore 0 & 0 & 0 & 1 & 0 \\
\hline Epinephelus merra & Carnivore 0 & 0 & 0 & 2 & 0 \\
\hline Epinephelus rivulatus & Carnivore 0 & 0 & 7 & 6 & 6 \\
\hline Epinephelus sexfasciatus & Carnivore 0 & 0 & 5 & 1 & 4 \\
\hline
\end{tabular}

\begin{tabular}{lllllll}
\hline Siganidae & Herbivore & 0 & 0 & 0 & 0 & 1 \\
Siganus argenteus & Herbivore & 0 & 7 & 0 & 0 & 0 \\
Siganus canaliculatus & Herbivore & 0 & 0 & 0 & 12 & 0 \\
Siganus guttatus & Herbivore & 0 & 3 & 0 & 16 & 0 \\
Siganus virgatus & Herbivore & 0 & 3 & 0 & 0 & 0 \\
Siganus vulpinus & Herbivore & 0 & 0 & 0 & 2 & 0 \\
Zanclidae & & & & & & \\
Zanclus cornotus & & & & & & \\
\hline
\end{tabular}

The composition of the five most diverse fish families that were observed in all sub-districts are given in Table 2. The most abundant families at sub district of Pari were Labridae (wrasses), followed by Pomacentridae (damselfishes). The most abundant families at the rest subdistricts were Pomacentridae (damselfishes), followed by the Labridae (wrasses). Overall, the most diverse families in the reef community were Pomacentridae and Labridae. This pattern was also observed in the previous study at the islands (Estradivari et al. 2007), and at other locations in Indonesia (Ferse 2008).

Table 2 The composition of the 5 most diverse fish families (\%)observed in all sub-districts

\begin{tabular}{llllll}
\hline Family & Pari & Tidung & Panggang & Kelapa & Harapan \\
\hline Apogonidae & 3.0 & - & 5.0 & 2.2 & 4.5 \\
Caesionidae & - & 4.8 & 7.8 & 6.0 & 6.4 \\
Chaetodontidae & - & 1.1 & 1.4 & - & - \\
Labridae & 59.8 & 30.5 & 24.7 & 23.9 & 24.8 \\
Nemipteridae & 0.6 & - & - & - & - \\
Pempheridae & 6.3 & - & - & - & - \\
Pomacentridae & 28.4 & 59.6 & 57.6 & 62.2 & 59.7 \\
Scaridae & - & 1.7 & - & 1.4 & 1.4 \\
\hline
\end{tabular}

The Shannon-Wiener diversity indices of the fish communities, the average species richness and the average fish abundance are shown in Figure 2. The sub-district Pari as the nearest to Jakarta Bay, had the lowest fish abundance, species richness, fish diversity, while the subdistrict Harapan as the outlier islands had the highest ones. The fish abundance ranged from $265 \pm 140$ (sub-district of Pari) to $1154 \pm 208 \mathrm{ind} / 250 \mathrm{~m}^{2}$ (sub-district of Harapan). Similar patterns were also found for the diversity indices $\left(\mathrm{H}^{\prime}\right)$ which ranged between $1.8 \pm 0.15$ (Pari) and $2.5 \pm 0.17$ (Harapan), and for the species richness which ranged from $20 \pm 7$ (Pari) to $36 \pm 5$ species $/ 250 \mathrm{~m}^{2}$ (Harapan) over the entire study period. The pattern showed that values on the fish abundance, diversity index and species richness increase toward north of Seribu Islands. The high abundance and species richness might be related to live coral coverage. The nearest region to Jakarta Bay has lowest live coral coverage and the live coral coverage increase toward to the north of the islands (Estradivari et al. 2007). In the present study, the sub-district's reefs did have a significant influence on fish abundance and species richness, but not for diversity index (Table 3). Multiple studies have reported a positive correlation with the structural complexity of a coral reef habitat for fish abundance (e.g. Walker et al. 2009), species richness (e.g. Wilson et al. 2007), and species diversity (e.g. Öhman and Rajasuriya 1998). 
Table 3 Results of Poisson regression for abundance, species richness, and diversity of fish assemblages $(*<0.001$, n.s. not significant)

\begin{tabular}{llllll}
\hline Variable & Factor df & W.S & p & \\
\hline Abundance & Site & 4 & 1868.0 & 0.00 & $*$ \\
Species richness & Site & 4 & 21.083 & 0.00 & $*$ \\
Shannon-Wiener index $\left(\mathrm{H}^{\prime}\right)$ Site & 4 & 0.50872 & 0.97 & n.s \\
\hline
\end{tabular}


Figure 2. The average values of (a) fish abundance, (b) species richness, and (c) species diversity (Shannon-Wiener Index; ln basis) of fish assemblages at the sampling sites. The arrow shows the direction from Jakarta Bay to the offshore water of the outer islands.

Besides being used as a territory (Waldner and Robertson 1980; Patton 1994), coral reefs are source of food for fishes (Reese 1981). The percentage of trophic level of total fish species at each sub-district is shown in Figure 3. The trophic level of species at each sub-district varied. The fish community in sub-district of Pari was dominated by carnivorous, omnivorous and herbivorous fishes, while those in the rest sub-districts were dominated by omnivorous and carnivorous fishes. Even though there is a strong correlation between coral and fish, only few of the species found in a coral reef ecosystem depend specifically on scleractinian corals (Munday et al. 2007). A study indicated that the fish communities were likely not structured by habitat-mediated factors such as predation impact or available space, but different factors such as recruitment or migration were playing a stronger role (Madduppa et al. 2012). However, no significant differences in diversity indices were found among the subdistricts (Table 3). This might be explained by feeding specialization among coral fishes. The specialization in food can reduce competition within a reef (Gladfelter and Johnson 1983; Ross 1986), and increase species diversity. A study found that some species such as scarids appeared in only specific habitat which had the lowest amount of live coral but the highest amount of dead coral and algae (Madduppa et al. 2012). Other species such as Chaetodonts have been observed to appear on high percentage of live coral which they use for food or shelter (Cox 1994).

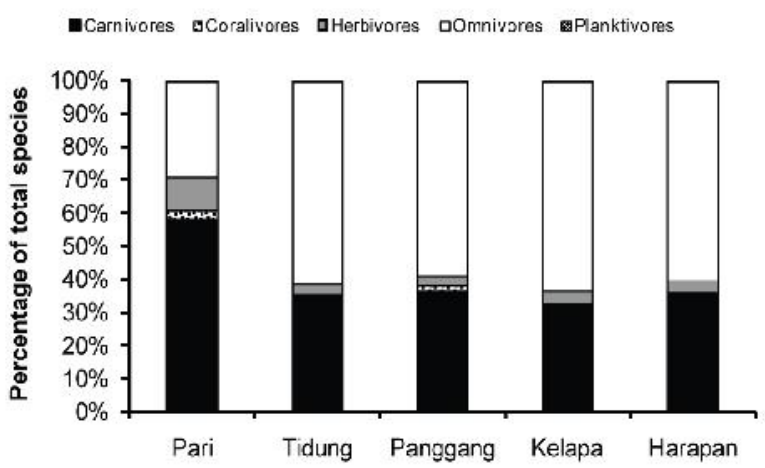

Figure 3. Distribution and mean composition of reef fish per sub districts at Seribu Islands based on trophic categories

The multivariate analysis of fish abundance, species richness and fish diversity were done using the Bray Curtis similarity index and non-metric multidimensional scaling (MDS). The MDS plot and Bray-Curtis similarity have distinctly clustered the sub-districts from southern and toward north of the Seribu Islands based on fish abundance, species richness and fish diversity. The results showed that the sub-districts can be clustered into 3 groups based on fish abundance, with 0 stress value (Figure 4). Group one consists of one sub-district (Pari) located in the southern part of the Seribu Islands near Jakarta Bay. Group two consists of three sub-districts (Tidung, Panggang, and Kelapa) located in mid of the archipelago. The third group consists of one sub-district (Harapan) located in the northern part of the Seribu Islands. The species richness and fish diversity indices showed that the sub-districts can be clustered into two groups (1=Pari and Tidung, 2= Panggang Kelapa, Harapan). These figures showed that the islands within archipelago seemed to be linked to the environmental factors such sedimentation, pollution and other human activities in Jakarta Bay and Seribu Islands (Rees et al. 1999; Rachello-Dolmen and Cleary 2007; Willoughby 1986). 


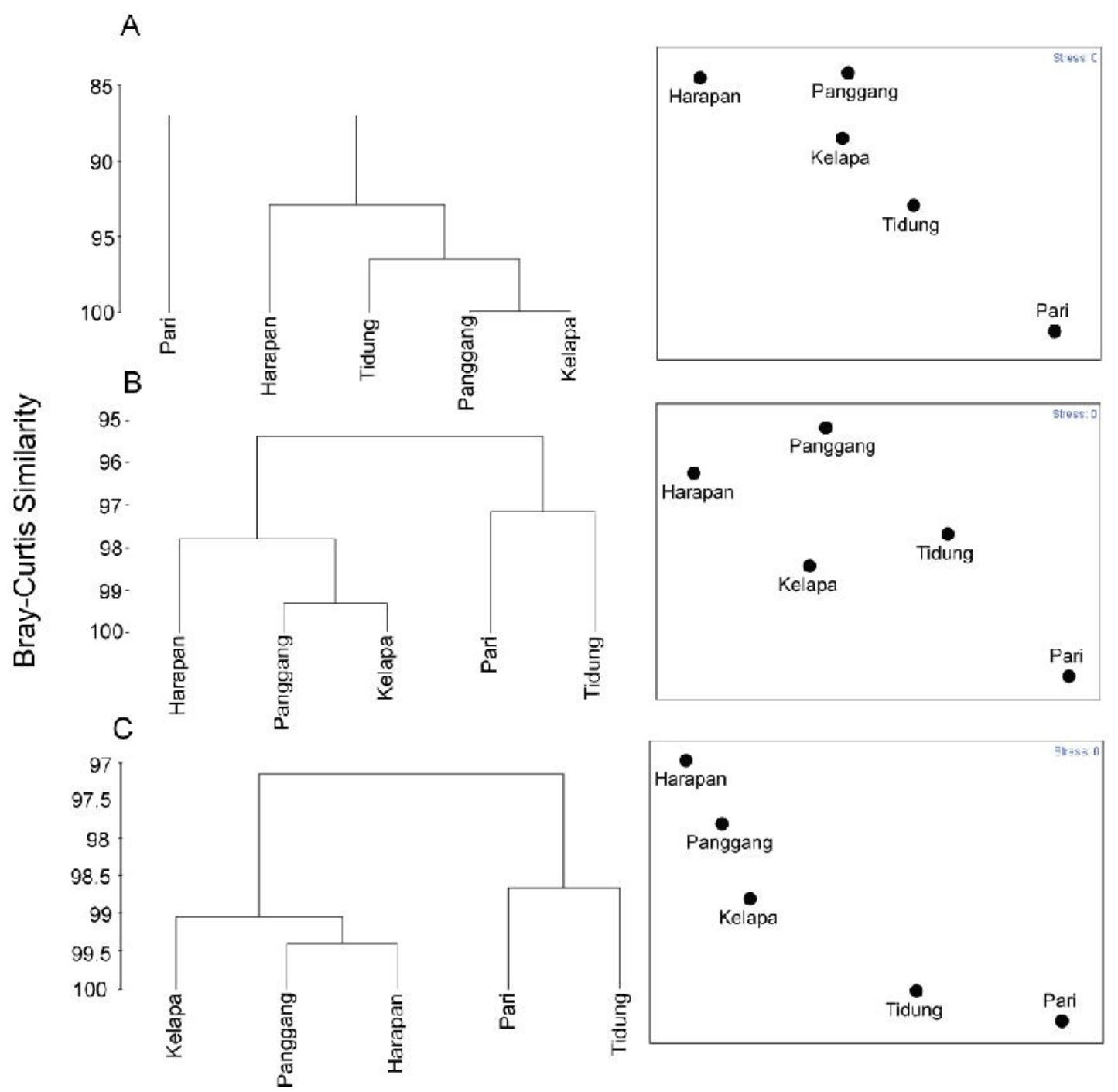

Figure 4. Dendogram based on Bray-curtis similarity (left) and MDS plot (right) of fish communities at the Seribu Islands, showing pattern of association among 216 species based on abundance (a), species richness (b) and fish diversity (c)

\section{CONCLUSION}

Altogether, in spite of low replicate of transects in each studied reefs, the present study results showed that the biodiversity of reef fishes across the Seribu Islands seems to be linked to environmental condition such as turbidity and level of pollution from Jakarta Bay toward the northern of the islands. Further studies of reef fish communities and habitat characteristics throughout the region are needed to document environmental changes over time.

\section{ACKNOWLEDGEMENTS}

We wish to thank the following institutions and people for their assistance and help during this study. Marine Biological Laboratory, Bogor Agricultural University (IPB) Bogor for the logistic support, members of Fisheries Diving Club, Bogor Agricultural University (IPB) Bogor for all their help in the field work. The study was supported by Agency of Fisheries and Marine Affairs, Government of DKI Jakarta.

\section{REFERENCES}

Allen GR. 2000. Marine fishes of South-East Asia. Periplus Editions (HK) Ltd, Singapore.

Allen GR, Werner TB. 2002. Coral Reef Fish Assessment in the 'Coral Triangle' of Southeastern Asia. Environ Biol Fish 65: 209-214.

Brock RE. 1982. A critique of the visual census method for assessing coral reef fish populations. Bul Mar Sci 32: 269-276.

Brown BE, Suharsono. 1990. Damage and recovery of coral reefs affected by El Niño related seawater warming in the Thousand Islands, Indonesia. Coral reefs 8: 163-170.

Burke L, Reytar K, Spalding M, Perry A. 2011. Reefs at Risk Revisited. World Resources Institute (WRI), Washington DC.

Clarke KR, Gorley NR. 2001. PRIMER v5: Unser manual/tutorial. PRIMER-E Ltd., Plymouth.

Clarke KR, Green RH. 1988. Statistical design and analysis for a 'biological effects' study. Mar Ecol Prog Ser 46: 213-226. 
Cleary DFR, Suharsono, Hoeksema BW. 2006). Coral diversity across a disturbance gradient in the Pulau Seribu reef complex off Jakarta, Indonesia. Biodiversity and Conservation. 15: 3653-3674.

Colton DE, Alevizon WS. 1981. Diurnal variability in a fish assemblage of a Bahamian coral reef. Environ Biol Fish 6: 341-345.

Cox EF. 1994. Resource use by Corallivorous Butterflyfishes (Family Chaetodontidae) in Hawaii. Bull Mar Sci 54: 535-545

de Voogd NJ, Cleary DFR. 2008. An analysis of sponge diversity and distribution at three taxonomic levels in the Thousand Islands/Jakarta Bay reef complex,West-Java, Indonesia. Mar Ecol 29: 205-215

English S, Wilkinson C, Baker V. 1997. Survey manual for tropical marine resources. Australian Institute of Marine Science (AIMS) Townsville.

Estradivari, Syahrir M, Susilo N, Yusri S, Timotius S. 2007. Coral Reef Jakarta: long-term observations of coral reefs Seribu Islands (2005 2007). Yayasan TERANGI, Jakarta. [Indonesian].

Ferse SCA. 2008. Artificial reef structures and coral transplantation: fish community responses and effects on coral recruitment in North Sulawesi/Indonesia. [Dissertation]. University of Bremen, Bremen, Germany.

Field JG, Clarke KR, Warwick RM. 1982. A practical strategy for analysing multispecies distribution patterns. Mar Ecol Prog Ser 8: $37-$ 52

Froese R, Pauly D. 2010. FishBase. World Wide Web electronic publication. www.fishbase.org

Gladfelter WB, Ogden JC, Gladfelter EH. 1980. Similarity and Diversity Among Coral Reef Fish Communities: A comparison between Tropical Western Atlantic (Virgin Islands) and Tropical Central Pacific (Marshall Islands) Patch Reefs. Ecology 61: 1156-1168.

Halford AR, Thompson AA. 1994. Visual census surveys of reef fish. Long-term monitoring of the Great Barrier Reef. Australian Institute of Marine Science (AIMS), Townsville.

Hoeksema BW. 1991. Control of bleaching in mushroom coral populations (Scleractinia: Fungiidae) in the Java Sea: stress tolerance and interference by life history strategy. Mar Ecol Prog Ser 74: 225 237.

Hughes TP, Baird AH, Bellwood DR, Card M, Connolly SR, Folke C, Grosberg R, Hoegh-Guldberg O, Jackson JBC, Kleypas J, Lough JM, Marshall P, Nystrom M, Palumbi SR, Pandolfi JM, Rosen B, Roughgarden J. 2003. Climate change, human impacts, and the resilience of coral reefs. Science 301: 929-933.

Hutomo M, Adrim M. 1985. Distribution of reef fish along transects in Bay of Jakarta and Kepulauan Seribu. In: Human induced damaged to coral reefs. Unesco Reports in Mar. Sci. 40: 135-156.

Kruskal JB. 1964. Multidimensional scaling by optimizing goodness of fit to a nonmetric hypothesis. Psychometrika 29: 1-27.

Kuiter RH. 1992. Tropical reef-fishes of the Western Pacific, Indonesia and adajacent waters. Gramedia, Jakarta.

Madduppa HH, Ferse SCA, Aktani U, Palm HW. 2012. Seasonal trends and fish-habitat associations around Pari Island, Indonesia: setting a baseline for environmental monitoring. Environ Biol Fish 95:383398.

Magurran AE. 1988. Ecological diversity and its measurement. Princeton University Press, Princeton.
Marques JC, Pardal MÂ, Nielsen SN, Jørgensen SE. 1997. Analysis of the properties of energy and biodiversity along an estuarine gradient of eutrophication. Ecol Modell 102, 155-167.

Munday PL, Jones GP, Sheaves M, Williams AJ, Goby G. 2007. Vulnerability of fishes on the Great Barrier Reef to climate change. In: Johnson J, Marshall P (eds) Climate change and the Great Barrier Reef. Great Barrier Reef Marine Park Authority, Townsville.

Öhman MC, Rajasuriya A. 1998. Relationships between habitat structure and fish communities on coral. Environ Biol Fish 53: 19-31.

Patton WK. 1994. Distribution and ecology of animals associated with branching corals (Acropora spp.) from the Great Barrier Reef, Australia. Bul Mar Sci 55:193-211

Rachello-Dolmen PG, Cleary DFR. 2007. Relating coral traits to environmental conditions in the Jakarta Bay/Pulau Seribu reef complex, Indonesia. Estuar Coast Shelf Sci 73: 816-826.

Reese JG, Setiapermana D, Sharp VA, Weeks JM, Williams TM. 1999. Evaluation of the impacts of land-based contaminants on the benthic faunas of Jakarta Bay, Indonesia. Oceanologica Acta 22: 627-640.

Renema W. 2008. Habitat selective factors influencing the distribution of larger benthic foraminiferal assemblages over the Kepulauan Seribu. Mar Micropaleontol 68: 286-298.

Ross ST. 1986. Resource partitioning in fish assemblages: a review of field studies. Copeia 1986:352-388

Shannon CE, Weaver W. 1949. The mathematical theory of communication. University of Illinois Press, Urbana.

Shepard R. 1962. The analysis of proximities: multidimensional scaling with an unknown distance function. II. Psychometrika 27: 219-246.

Tomascik T, Mah A J, Nontji A, Moosa MK. 1997. The Ecology of the Indonesian Seas, Part One and Two. Periplus, Hongkong.

Uneputty PA, Evans SM. 1997. Accumulation of beach litter on Islands of the Kepulauan Seribu Archipelago, Indonesia. Mar Poll Bull 34 (8): 652-655.

van der Meij SET, Moolenbeek RG, Hoeksema BW. 2009. Decline of the Jakarta Bay molluscan fauna linked to human impact. Mar Poll Bull 59: 101-107.

Verstappen HTh. 1988. Old and new observations on coastal changes of Jakarta Bay: an example of trends in urban stress on coastal environments. J Coast Res 4 (4): 573-587.

Waldner RE, Robertson DR. 1980. Patterns of habitat partitioning by eight species of territorial Caribbean damselfishes (Pisces: Pomacentridae). Bul Mar Sci 30:171-186

Walker BK, Jordan LKB, Spieler RE. 2009. Relationship of Reef Fish Assemblages and Topographic Complexity on Southeastern Florida Coral Reef Habitats. J Coast Res 39-48.

Williams TM, Rees JG, Setiapermana D. 2000. Metals and trace organic compounds in sediments and waters of Jakarta Bay and the Pulau Seribu Complex, Indonesia. Mar Poll Bull 40 (3): 277-285.

Willoughby NG. 1986. Man-made litter on the shores of the Thousand Island Archipelago, Java. Mar Poll Bull 17: 224-228.

Wilson S, Graham N, Polunin N. 2007. Appraisal of visual assessments of habitat complexity and benthic composition on coral reefs. Mar Biol 151: 1069-1076.

Zaneveld JS, Verstappen HTH. 1952. A recent investigation about the geomorphology and the flora of some coral islands in the Bay of Djakarta. J Sci Res 3: 58-68 